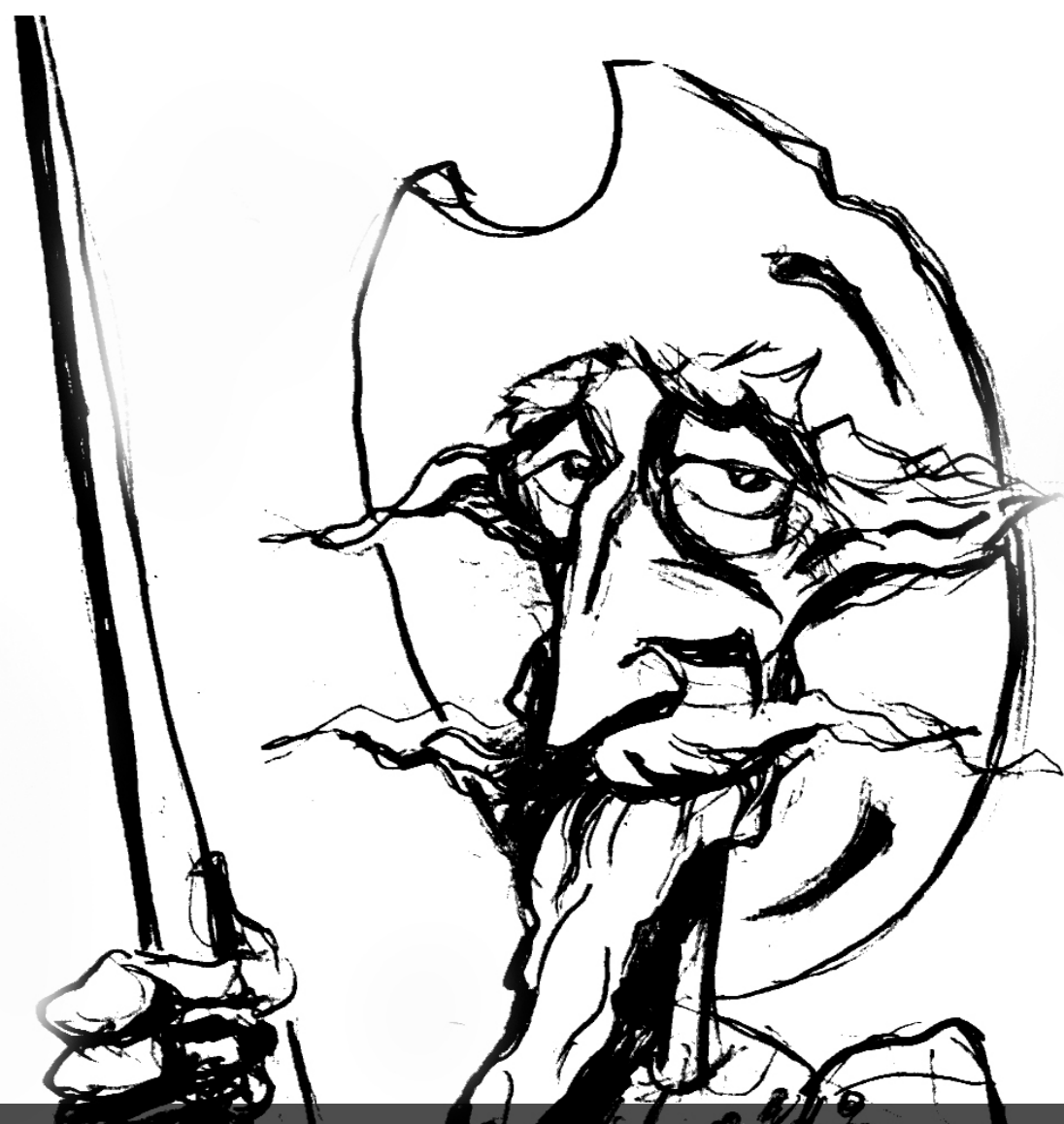

La Sierra de Santiago y el Valle de Catamarca (Gobernación del Tucumán) a principios del siglo XVII: reflexionando sobre una nueva regionalización

[Isabel Castro Olañeta y Leticia Carmignani] 


\title{
La Sierra de Santiago y el Valle de Catamarca (Gobernación del Tucumán) a principios del siglo XVII: reflexionando sobre una nueva regionalización *
}

\section{The Mountains of Santiago and the Catamarca Valley (Governorate of Tucuman) in the Early Seventeenth Century: Reflecting on a New Regionalization}

\author{
ISABEL CASTRO OLAÑETA \\ LETICIA CARMIGNANI
}

\begin{abstract}
Resumen
Presentamos el análisis de un informe fechado en febrero de 1607, resultado de la Visita al Partido de la Sierra-dependiente y parte de la jurisdicción de la ciudad de Santiago del Estero-, que fue realizada por el Teniente de Naturales Francisco de Betanzos quien, por orden del Gobernador del Tucumán Alonso de Ribera, recorrió la tierra aplicando las Ordenanzas de Abreu y desagraviando a los indios. La visita incluye información sobre el valle de Catamarca y la ladera oriental del cordón Ancasti y Alto-Gracián, las sociedades indígenas y el funcionamiento de las encomiendas. Su análisis nos permite proponer una nueva regionalización para la Gobernación del Tucumán y una posible complementariedad económica entre sus distintos espacios aprovechada por los encomenderos en el período temprano colonial.
\end{abstract}

Palabras clave

Colonia temprana - Santiago del Estero - Valle de Catamarca - Región - Encomiendas

\begin{abstract}
We present here the analysis of a report dated on February 1607, as a result of the Visit to the district of the Sierra-dependent and part of the jurisdiction of the City of Santiago del Estero, which was conducted by the "Teniente de Naturales" Francisco de Betanzos, who, by order of Governor of Tucuman Alonso de Ribera, visited the land and applied the Abreu's Ordinances to make reparations to the Indians. The visit includes information about Catamarca Valley and the eastern hillside of Ancasti and Alto-Gracian mountains, the indigenous societies and the "encomiendas". This analysis allows us to propose a new regionalization for Governorate of Tucuman and a possible economic complementarity of the spaces used by the "encomenderos" in the early colonial period.
\end{abstract}

Key words

Early colonial period - Santiago del Estero Catamarca Valley - Región - Encomiendas

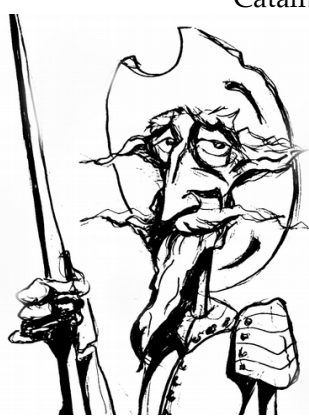

Recibido con pedido de publicación el 20 de febrero de 2017

Aceptado para su publicación el 25 de abril de 2017

Versión definitiva recibida el 11 de mayo de 2017

Isabel Castro Olañeta, Universidad Nacional de Córdoba, Argentina; e-mail: isabelcastrox@gmal.com

Leticia Carmignani, Consejo Nacional de Investigaciones Científicas y Técnicas, Universidad Nacional de Córdoba, Argentina; e-mail: leticiacarmignani@gmail.com

* Las autoras agradecen los comentarios y sugerencias de los evaluadores anónimos de la Revista.

Esta obra se publica bajo licencia Creative Commons. Atribución-NoComercial-CompartirIgual Internacional

Castro Olañeta, Isabel; Carmignani, Leticia "La Sierra de Santiago y el Valle de Catamarca (Gobernación del Tucumán) a principios del siglo XVII: reflexionando sobre una nueva regionalización”, Prohistoria, Año XX, núm. 27, jun. 2017, pp. 522 .

\section{Introducción}

El proceso de exploración e invasión de la región del Tucumán se inicia con las primeras entradas e incursiones al sur del Kollasuyu en las décadas de 1530 y 1540 y continúa con la fundación y poblamiento de ciudades durante las décadas siguientes. Este proceso se da en medio de disputas jurisdiccionales que terminan definiendo en 1563 a la región como Gobernación del Tucumán - 
con cabecera en Santiago del Estero-, la cual formaba parte del virreinato del Perú y era dependiente en lo judicial de la Audiencia de Charcas.

Paralelamente al proceso fundacional los conquistadores se iban trasformando en vecinos encomenderos e iban organizando empresas productivas con base en sus encomiendas estimulados por la demanda y arrastre mercantil del polo minero potosino. Así, bajo el sistema del "servicio personal" utilizaban -casi ilimitadamente- la mano de obra indígena para poner en producción las tierras y generar -en una región no minera- excedentes exportables que les permitieron insertarse en el mercado peruano. ${ }^{1}$

En palabras de Assadourian, los vecinos encomenderos del Tucumán, aunque mostraban rasgos señoriales, no conformaban una aristocracia ociosa. Más bien, podrían ser caracterizados como comerciantes, minoría dirigente y empresarios, en el contexto de una economía de cambio. Es decir que estos conquistadores devenidos en encomenderos tenían una participación económica activa que posibilitó en un primer momento la creación de una economía de autosubsistencia mediante el uso de la fuerza laboral indígena y la introducción de plantas y animales europeos; pero que, a corto plazo, permitió que las ciudades contaran con abundantes productos de la tierra disponibles para ser mercantilizados y comercializados en los circuitos activados por el centro minero potosino. ${ }^{2}$

Por los altos niveles de explotación de los indios bajo el sistema de servicios personales y por la ausencia de funcionarios que controlaran a los encomenderos y castigaran sus abusos, además de las pestes y los efectos desestructuradores de la invasión española, la región sufrió una fuerte y continua caída demográfica que tuvo uno de sus puntos máximos en la década de 1590. Esta situación era conocida por la Corona quien remitió a la Audiencia de Charcas la orden de visitar la tierra, sin embargo, dicha visita se venía postergando por diversas razones demorándose el desagravio de las sociedades indígenas. Si bien la Visita llegó finalmente de la mano del oidor de la Audiencia Francisco de Alfaro en 1611, en los años previos el gobernador del Tucumán Alonso de Ribera intentó llevar adelante una serie de medidas favorables a los indios y contrarias a los encomenderos, entre las que se encontraba realizar una Visita de los indios de encomienda.

Entre 1606 y 1607 el Gobernador del Tucumán Alonso de Ribera designó lugartenientes denominados Tenientes de Naturales con la atribución de visitar, empadronar y ejecutar las Ordenanzas de Gonzalo de Abreu (1576), con el objetivo de desagraviar a los naturales que sufrían excesos y maltratos por parte de los encomenderos españoles y sus pobleros. Para ello, les confirió jurisdicción civil y criminal para poder aplicarla al interior de los partidos de pueblos de indios, que eran distritos menores dentro de la jurisdicción de las ciudades, pero de territorialidad indígena, que él mismo delimitó. Mientras duraron en sus cargos (ya que, a poco de comenzar la Visita, por presión de los vecinos encomenderos del Tucumán llegó una orden de la Audiencia de Charcas de suspenderla), los Tenientes de Naturales empadronaron a los indios, recogieron denuncias, levantaron causas $y$ castigaron a pobleros $y$

\footnotetext{
${ }^{1}$ ASSADOURIAN, Carlos Sempat "La conquista", en ASSADOURIAN, C. S., BEATO G. y J. C. CHIARAMONTE, J. C. Argentina: De la conquista a la independencia, Hyspamérica, Buenos Aires, 1986 [1972]. PALOMEQUE, Silvia "El mundo indígena. Siglos XVI-XVIII", en TANDETER, Enrique (dir.) Nueva Historia Argentina II, La sociedad Colonial, Editorial Sudamericana, Buenos Aires, 2000.

2 ASSADOURIAN, Carlos Sempat “La conquista...”, cit., pp. 93-97.
} 
encomenderos; como resultado elaboraron informes y numeraciones sobre estas visitas y actuaciones en sus distritos a cargo y se los presentaron al gobernador. Hasta el momento, de estos documentos conocíamos solo los resúmenes que hizo el Gobernador para remitir a la Corona y una Numeración de indios del Partido del Río Salado.

En este trabajo, que continua investigaciones previas de ambas autoras ${ }^{3}$, nos proponemos recuperar lo que consideramos un importante documento de 1607 -el Informe original elevado al Gobernador Ribera por parte del Teniente de Naturales designado para el Partido de la Sierra-, la información brindada por el mismo y su contexto de producción ${ }^{4}$ y aportar un primer análisis del documento que incluya una reconstrucción del espacio, las características del territorio, las encomiendas y empresas productivas españolas de la región, su relación con la ciudad de Santiago del Estero y con otros partidos.

El Informe fechado en Alivigasta, en 7 de febrero de 1607 incluye descripciones geográficas, de las sociedades indígenas, del funcionamiento de las encomiendas, así como un detalle de su propio recorrido a lo largo de la visita. Podemos afirmar que su autor, Francisco de Betanzos, fue uno de los Tenientes designados por Alonso de Ribera por la fecha del documento, porque está dirigido al Gobernador y porque claramente alude a su función de empadronar y aplicar las Ordenanzas de Gonzalo de Abreu sobre su "distrito".

El recorrido de este Teniente comprende tanto el valle de Catamarca (perfectamente distinguido y separado del de Londres), como el espacio conocido como la "Sierra de Santiago" y que se correspondía con la vertiente oriental del cordón Alto-Gracián-Ancasti que cae hacia la planicie del Río Dulce y que integra la suave elevación de Guasayán. Esta distinción nos permite sostener que el Partido de la Sierra delimitado por el Gobernador Alonso de Ribera para la visita incluía no solo la sierra de Guasayán, sino también el altoGracián-Ancasti, su piedemonte oriental e incluso, el valle de Catamarca.

El artículo se estructura en cuatro partes. En primer lugar, comienza con la síntesis del proceso de nombramiento de los Tenientes de Naturales por parte del Gobernador Alonso de Ribera y la definición de sus atribuciones; en segundo lugar se analiza la delimitación de distritos o "partidos de pueblos de indios" los sobre los cuales realizarían sus visitas; en tercer lugar, se presenta un análisis detallado del documento de 1607 para concluir que se trata del extraviado Informe del Teniente de Naturales que tenía a su cargo el Partido de la Sierra y, en cuarto lugar, nos detendremos en el análisis que, sobre el espacio

\footnotetext{
${ }^{3}$ CARMIGNANI, Leticia "Las visitas de los Tenientes de Naturales a los partidos de pueblos de indios de la Gobernación del Tucumán (1606-1607)", Corpus. Archivos virtuales de la alteridad Americana, vol. 3, núm. 2, 2013, Julio/Diciembre 2013: http: / / corpusarchivos.revues.org/548 y http:/ / corpusarchivos.revues.org/557

CARMIGNANI, Leticia "'Soy en esta provincia muy odiado de los vecinos encomenderos'. El Gobernador del Tucumán Alonso de Ribera y su proyecto político (1605-1611)", Memoria Americana. Cuadernos de Etnohistoria, vol. 23, núm. 1 (2015), Sección Etnohistoria, Facultad de Filosofía y Letras-UBA, Buenos Aires, enero-junio 2015, pp. 11-38.

http://ppct.caicyt.gov.ar/index.php/memoria-americana/article/view/5862/6183

CASTRO OLANETA, Isabel "La Numeración de los indios del partido del Río Salado. Santiago del Estero, 1607. Encomiendas y servicio personal", Corpus. Archivos virtuales de la alteridad Americana, vol. 3, núm. 2, 2013, Julio/Diciembre 2013: http: / / corpusarchivos.revues.org/535 y http:// corpusarchivos.revues.org/625

${ }^{4}$ La transcripción paleográfica del "Informe sobre la visita al Partido de la Sierra y valle de Catamarca, enviado por el Teniente de Naturales Francisco de Betanzos al Gobernador del Tucumán, Alonso de Ribera. Alivigasta, 7 de febrero de 1607" (ABNB, CACh, 580), de las mismas autoras en este mismo número de la Revista.
} 
visitado, nos brinda el Informe. Finalmente, analizaremos la posible complementariedad productiva entre las encomiendas y mercedes territoriales situadas en el valle de Catamarca con las de otros partidos y jurisdicciones entre fines del siglo XVI y principios del siglo XVII, avanzando en una propuesta de regionalización del espacio colonial temprano del Tucumán. Esto lo haremos en el marco del planteo de Darío Barriera quien entiende que el territorio colonial no solo debe ser pensado a partir de la delimitación de jurisdicciones a priori desde los poderes centrales, sino que los actores locales y regionales son los que irán configurando el proceso de espacializacion de dicho territorio a partir de diversos factores. ${ }^{5}$

\section{Los Tenientes de Naturales}

El informe que describe el recorrido de la visita "del valle de Catamarca a esta sierra" debe ser comprendido como parte del proyecto y las políticas llevadas adelante por el Gobernador del Tucumán Alonso de Ribera (1605-1611) quien asume su cargo en el contexto de una gran caída demográfica de las sociedades indígenas a causa de los abusos, excesos, malos tratos y altísimos niveles de explotación a los que estaban sometidos los indígenas, en el marco de la encomienda de servicios personales. Sabemos que la encomienda de servicios personales era en esta región la institución que permitía al español encomendero disponer de la mano de obra indígena de manera casi ilimitada, en tanto la materia y la cantidad del tributo no se encontraban tasadas, sino que solo se regulaba el tiempo de trabajo. ${ }^{6}$

Si bien esta situación de explotación venía siendo denunciada por los grupos religiosos y otros actores de la sociedad colonial, recién en los primeros años del siglo XVII, con la gestión de Alonso de Ribera estas denuncias se articulan en torno a su proyecto político que buscaba, en principio, remediar la situación utilizando el marco normativo vigente de las ordenanzas de Abreu de 1576, mientras pensaba reformarlas a futuro y además proyectaba tasar los tributos. $^{7}$ En este contexto y a partir del nombramiento de funcionarios específicos, el Gobernador Ribera llevó adelante un proyecto de "visita de la tierra" de las sociedades indígenas encomendadas, con el objeto de "desagraviarlas" frente a los excesos y malos tratos que sufrían por parte de los encomenderos españoles y sus pobleros. ${ }^{8}$

\footnotetext{
${ }^{5}$ BARRIERA, Darío Abrir puertas a la tierra. Microanálisis de la construcción de un espacio político, Santa Fe, 1573-1640, Ministerio de Innovación y Cultura de la Provincia de Santa Fe, Museo Histórico Provincial Brigadier Estanislao López, Santa Fe, 2013, pp. 32-34.

6 PALOMEQUE, Silvia "El mundo indígena...", cit., pp. 87-143; DOUCET, Gastón "La encomienda de servicio personal en el Tucumán, bajo régimen legal: comentarios a las ordenanzas de Gonzalo de Abreu", en LEVAGGI, Abelardo (coord.) El aborigen y el derecho en el pasado y el presente, Universidad del Museo Social Argentino, Buenos Aires, 1990, pp. 141-244; CASTRO OLAÑETA, Isabel "La Numeración...", cit.

${ }^{7}$ Sobre las denuncias acerca de la situación de explotación: DOUCET, Gastón "Génesis de una 'visita de la tierra': los orígenes de la visita de las gobernaciones de Tucumán y Paraguay por el Licenciado Don Francisco de Alfaro", Revista de Historia del Derecho, núm. 14, pp. 123-220; CROUZEILLES, Carlos Religiosos y sociedad colonial. Los religiosos, su ingreso al Tucumán y su incidencia politica en el sistema de explotación económico sobre la sociedad indígena (Tucumán, 15351615), Ferreyra Editor, Córdoba, en prensa.

${ }^{8}$ Este apartado y los siguientes son una síntesis de una investigación sobre el proyecto político y gestión del Gobernador Alonso de Ribera (1605-1611), CARMIGNANI, Leticia "'Soy en esta provincia..." cit.
} 
Así, la medida principal del gobernador, y parte central de su proyecto de gobierno, fue el nombramiento entre 1606 y 1607, de Tenientes de Naturales inspirados en los corregidores de indios del Perú, es decir, como funcionarios coloniales con atribuciones para imponer el poder del estado colonial al interior del territorio indígena. ${ }^{9}$ Los Tenientes de Naturales tenían orden de visitar la tierra, desagraviar a los indios y castigar los abusos ejecutando las Ordenanzas vigentes, y para ello el Gobernador les concedió funciones y atribuciones que los asemejaban a los corregidores de indios: les confirió jurisdicción civil y criminal otorgándoles "bara alta de la real justicia" para aplicar las ordenanzas de Abreu del 1576, delegándoles la facultad de hacer "que los encomenderos y sus pobleros las guarden y cumplan" y, para ello, les delimitó y asignó jurisdicciones para la realización de la visita denominadas partidos de pueblos de indios. Debían también controlar que "los casiques e yndios les acudan con lo que deben y son obligados conforme a ellas", es decir que tributaran según la tasa de tiempo de trabajo ordenada por Abreu, "sin consentir ni dar lugar a que [a los indios] se les haga molestia ni agravio [...] castigando con demostracion a los que se los hizieren". Estos funcionarios podían actuar en "cualesquier pleitos y causas cibiles y criminales" pudiendo iniciar causas contra cualquier persona, incluyendo pobreros, encomenderos e indios. Especialmente, debían aplicar justicia ante los delitos referentes a los "muchos daños de malos tratamientos y excesivos castigos con demasiada crueldad y haziendolos trabajar de dia y de noche y los dias de fiestas y domingos sin les dar de comer."10

Dentro de las tareas que tenían que realizar los tenientes, según se describe en sus nombramientos, se incluía la realización de numeraciones o padrones de la población indígena encomendada y sabemos que, al menos en el Partido del Río Salado, se había alcanzado a elaborar el padrón de indios que Alonso de Ribera adjunta y envía al Rey. ${ }^{11}$ La realización de los padrones se vincula al proyecto inconcluso del Gobernador de tasar el tributo que los indios debían entregar -como en las regiones centrales del virreinato-, tasación que recién llegó con la Visita y Ordenanzas de Francisco de Alfaro en 1611-1612.

Sin embargo, este proyecto y específicamente la medida de nombramiento de los Tenientes fue anulada por la Audiencia de Charcas en 1607 que le ordenó "no innovar en el gobierno", y así las visitas debieron ser suspendidas a poco tiempo de haberse comenzado y no se pudo tasar el tributo ni modificar las ordenanzas. Si bien esta suspensión dejó incompleto el proyecto de Ribera, consideramos dicha medida como el mayor embate de este Gobernador contra el sector encomendero local ya que atacó su capacidad de hecho de beneficiarse sin controles de la mano de obra indígena. Su preocupación por la conservación de las sociedades indígenas, significó la intervención en las encomiendas de un funcionario colonial denominado Teniente de Naturales con atribuciones para aplicar justicia y desagraviar a las sociedades indígenas.

\footnotetext{
${ }^{9}$ ASSADOURIAN, Carlos Sempat El sistema de la economía regional: mercado interno, regiones y espacio económico, Instituto de Estudios Peruanos, Lima, 1982, p. 306.

${ }^{10}$ Se conservan las copias de los Títulos o Nombramientos de los Tenientes nombrados para los partidos de Córdoba en las Actas Capitulares de dicha ciudad. Rescatamos aquí la Copia del Título de don Luciano de Figueroa, del 16 de diciembre de 1606. Transcripta en CARMIGNANI, Leticia "Las visitas de los Tenientes...", cit., pp.24-27.

${ }^{11}$ El padrón del Partido del Río Salado, transcripto en: CASTRO OLAÑETA, Isabel "La Numeración de los indios...", cit. También contamos con referencias de que el padrón fue realizado en el Partido de Soto, ver: PIANA, Josefina Los indígenas de Córdoba bajo el régimen colonial 1570-1620, Córdoba, 1992, p. 256.
} 


\section{Los partidos de pueblos de indios}

Como ya hemos adelantado, estos Tenientes de Naturales, entre 1606 y 1607, visitaron la Gobernación del Tucumán aplicando las ordenanzas de Abreu, cada uno sobre una circunscripción territorial determinada y denominada partido de pueblos de indios, dependiente y parte de la jurisdicción de las ciudades. ${ }^{12}$ La territorialidad -rural e indígena- de los partidos incluía dos tipos de asentamientos: pueblos de indios de encomienda y núcleos productivos españoles como estancias u obrajes beneficiados con la mano de obra indígena.

Si bien por haber sido suspendidas las visitas no se logró el empadronamiento o numeración de los indios de todos los partidos $-y$ mucho menos la tasa-, los Tenientes de Naturales elaboraron relaciones o informes al Gobernador acerca de lo observado y lo resultado en la aplicación de la justicia en cada jurisdicción. Dichos informes, además de confirmar la efectiva realización de las visitas y de dar cuenta de los maltratos y excesos que sufrían los indios, ofrecen valiosa información acerca de la situación y características del servicio personal en los partidos de cada jurisdicción. Con respecto a la información que incluyen y cómo la presentan, los informes varían entre sí, ya que algunos Tenientes exponen de manera general los agravios que "iban remediando", mientras otros enumeran detalladamente las causas judiciales iniciadas contra encomenderos y pobleros. En síntesis, los informes constituyen una excelente fuente para acercarse al funcionamiento del sistema de la encomienda de servicios personales legalizado por Abreu y sus diferencias regionales. ${ }^{13}$

A su vez, estos informes sobre las visitas, permiten percibir el proceso de espacialización que se va dando en las diferentes regiones, en tanto el accionar del Gobernador -con su delimitación de distritos dentro de una jurisdicción mayor-, de los Tenientes -que recorren, aplican justicia, describen-, y de encomenderos y pobleros -que ponen en producción sus empresas agrarias con la mano de obra indígena-, nos permiten pensar que luego de la fundación de las primeras ciudades, el territorio va adquiriendo una lógica particular en diálogo con estos agentes. Además, las descripciones de los Tenientes sobre los ambientes y sus recorridos nos permiten reflexionar acerca del uso y la

\footnotetext{
12 "Partido" entendido como "el distrito o territorio que está comprendido de alguna jurisdicción o administración de una Ciudad Principal, que se llama su cabeza" (Real Academia Española Diccionario de la lengua castellana, en que se explica el verdadero sentido de las voces, su naturaleza y calidad, con las phrases o modos de hablar, los proverbios o refranes, y otras cosas convenientes al uso de la lengua [...]. Compuesto por la Real Academia Española. Tomo quinto. Que contiene las letras O.P.Q.R. Imprenta de la Real Academia Española, Madrid, 1737, pp. 141-142)

${ }^{13}$ Ejemplo de esto son los trabajos de QUIROGA, Laura "Las granjerías de la tierra: actores y escenarios del conflicto colonial en el valle de Londres (Gobernación del Tucumán, 1607-1611)", Surandino Monográfico, segunda sección del Prohal Monográfico, Vol. II, núm. 2, Buenos Aires, 2012, pp. 1-37; y CASTRO OLAÑETA, Isabel "'Donde estan situados los mas yndios de la jurisdiccion desta ciudad'. Un acercamiento etnohistórico a las encomiendas y pueblos de indios del Río Salado. Santiago del Estero entre fines del siglo XVI y principios del siglo XVII", Surandino Monográfico, vol. 3, núm. 2, 2013, pp, 1-23. http://revistascientificas.filo.uba.ar/index.php/prohals/article/view/477; CARMIGNANI, Leticia "Los 'partidos' de la jurisdicción de Córdoba, las sociedades indígenas y la percepción del espacio. Gobernación del Tucumán, principios del siglo XVII", en ROCCHIETTI Ana María, YEDRO Mariano y OLMEDO Ernesto (comps) Arqueología y Etnohistoria de Investigadores del Centro - Oeste Argentino. Publicación de las IX Jornadas de Investigadores en Arqueología y Etnohistoria del Centro - Oeste del país, Editorial UNIRIO, Universidad Nacional de Río Cuarto, Río Cuarto, 2013, pp. 161-172.
} 
construcción del territorio, de las formas de producción y de circulación que los actores locales y regionales van definiendo, más allá de la consolidación administrativa y jurisdiccional. Es decir que la Corona proyectaba cartografías administrativas que muchas veces eran diferentes al real ejercicio de las jurisdicciones, aunque sin atentar contra estas, más bien esas diferencias "definían siluetas de la territorialidad en función de los alcances de la potestad, lo que casi siempre daba una forma diferente a la prevista."14

Podemos conocer por las cartas del Gobernador al Rey, que para la jurisdicción de la ciudad de Santiago del Estero, Ribera designó Tenientes para tres partidos (Partido del río Dulce; del Río Salado y de la Sierra), para La Rioja dos partidos (Valle de Londres y de Famatina); para la ciudad de Córdoba tres (Partido de los Ríos; de Soto y de Quilino y Totoral) y para San Miguel de Tucumán suponemos que habría designado un solo partido (Partido de la Ciudad de San Miguel). Igualmente había nombrado Tenientes para las ciudades de Salta, Jujuy y Villa Madrid de las Juntas pero que no alcanzaron a realizar las visitas antes de la orden audiencial de acabar con ellas.

\section{El Informe de Francisco de Betanzos de 1607}

Hasta el momento, los informes de los Tenientes que conocíamos eran aquellos que fueron incorporados de mano del Gobernador Alonso de Ribera en sus cartas al Rey, presentando la información resumida o textual y, en un caso, incorpora un padrón de indios (el del Río Salado).Para algunos partidos, Ribera da el nombre de los Tenientes que realizaron los informes a los que refiere y para otros no. Entendemos que el Informe que aquí recuperamos, firmado por el Teniente Francisco de Betanzos, correspondería a esta serie de visitas y sería el primer Informe original (y no copiado o resumido por Ribera) al que es posible acceder de los Tenientes de Naturales que visitaron la Gobernación del Tucumán.

Si bien no hemos encontrado el nombramiento Francisco de Betanzos como Teniente de Naturales, contamos con varios indicios que nos permiten afirmar que lo fue. ${ }^{15}$ Por un lado, envía al Gobernador el informe con fecha de 7 de febrero de 1607, por lo que su actuación se ubica dentro del periodo en que los Tenientes de Naturales estaban visitando la tierra. ${ }^{16}$ Por otra parte, en el documento que nos ocupa, Francisco Betanzos informa que está realizando una visita y que en ella, como el Gobernador lo manda, va "aciendo padron y dandoles a entender lo que Vuestra Señoria manda por las ordenanças del governador Gonçalo de Avrego y Figueroa y como an de acudir a servir y que mi venida a sido para anparallos en justicia". Por esto, entendemos que el nombramiento de este funcionario debe haber sido similar a los de los Tenientes de Naturales que conocemos para Córdoba y que se le otorgaron sus mismas atribuciones, mientras se le asignaba y delimitaba un "partido de pueblo de indios", ya que él mismo informa haber "dado buelta a mi distrito".

\footnotetext{
${ }^{14}$ BARRIERA, Darío Abrir puertas..., cit., p. 32.

${ }^{15}$ En caso de haber existido dicho nombramiento por parte del Gobernador, entendemos que debería haber estado incorporado en las Actas de 1606 del cabildo de la ciudad de Santiago del Estero (tal como se han conservado los de Córdoba); o su copia en los expedientes que los procuradores de las ciudades elevaron a la Audiencia de Charcas protestando contra la medida de Alonso de Ribera de designar "corregidores encubiertos" con el título de "Tenientes de Naturales".

${ }^{16}$ Por ejemplo, para Córdoba, las visitas se realizaron entre el 29 de diciembre de 1606 y 14 de mayo de 1607.
} 
El informe analizado no especifica de manera puntual sobre cuál "partido de pueblos de indios" está realizando la visita el Teniente Betanzos, pero nos brinda los indicios suficientes para develarlo al contrastar la información ofrecida por el Teniente con otros documentos como son las cartas del Gobernador al Rey. En primer lugar, el 14 de mayo de 1607 -la carta de Betanzos es del 7 de mayo- el Gobernador informa sobre las visitas en los términos de la ciudad de Santiago del Estero, comprendiendo sus tres partidos: del Río Dulce; del Río Salado y de la Sierra; sin embargo, la información resumida es general y no da los nombres de los Tenientes de cada partido. ${ }^{17}$

También en la misma carta del 14 de mayo de 1607 incluye los resultados de las visitas de la jurisdicción de La Rioja, a la cual le había asignado dos partidos pero, en este caso, indicando los nombres de los Tenientes que las llevaron adelante: el Partido del Valle de Londres, a cargo del licenciado Jusepe de Fuensalida Menesses y el Partido de Famatina, visitado por el capitán Sebastián Guerrero. En este apartado, el Gobernador enumera y describe procesos judiciales que habían sido realizados contra encomenderos y pobleros indicando los pueblos de indios en donde habían tenido lugar determinados delitos. Así, si bien en un primer momento sospechamos que el Informe de Francisco de Betanzos sobre el Valle de Catamarca podría corresponder a otro Partido de La Rioja, la carta del Gobernador Ribera no da ningún indicio que nos haga pensar su adscripción a La Rioja, sino por lo contrario, el Gobernador detalla muy bien las visitas de Londres y Famatina como si no tuviera nada más para adjuntar ni explicar sobre la jurisdicción.

De la misma manera, para el caso de los partidos de la jurisdicción de la ciudad de Córdoba, Ribera en la misma carta, incorpora relaciones de los tres Tenientes que los visitaron: Luciano de Figueroa, Teniente del Partido de los Ríos, Juan de Betanzos de Quilino y Totoral y Francisco de Avellaneda del Partido de Soto, excluyendo la posibilidad de que existiera otro partido en esta jurisdicción. ${ }^{18}$

También indagamos si este distrito que incluía el valle de Catamarca podía corresponder a un Partido de la ciudad de San Miguel. Sabemos que en la carta del 14 mayo de 1607 el Gobernador avisa que de "los Tenientes de Esteco y de Salta y de las Juntas y San Miguel de Tuquman no an llegado con sus relaçiones

\footnotetext{
${ }_{17}$ Carta de Alonso de Ribera al Rey del 14 de mayo de 1607. AGI, CHARCAS, 26,R.8,N.46. (Transcripción completa en CARMIGNANI, Leticia "Las visitas de los Tenientes...", cit., pp. 113)

${ }^{18}$ En un primer momento, cuestionamos si, debido a la coincidencia en el apellido de Francisco y Juan de Betanzos, no se podría hablar de un mismo Teniente, considerando además la cercanía y conexión entre la región de Quilino y el valle de Catamarca; sin embargo, al revisar con cuidado la paleografía de ambos documentos, no cabe duda de que se trataría de dos personas distintas, aunque no estamos en condiciones de saber si estaban emparentados. A esta diferenciación además la podemos sustentar contrastándola con otras referencias. Sobre Francisco de Betanzos, podría suponerse que es el mismo capitán que estuvo al mando del Fuerte de Nacimiento (fundado el 24 de diciembre de 1603 por Alonso de Ribera) en la confluencia de los ríos Bio Bio y Vergara, en la frontera del Arauco. (GONZÁLEZ DE NÁJERA, Alonso "Desengaño y reparo de la guerra del reino de Chile", en Colección de Documentos Inéditos para la Historia de España, Tomo XLVIII, Imprenta de la Viuda de Calero, Madrid, 1866 (1614), p. 341) Alonso de Ribera, luego de su gestión como Gobernador en Chile a cargo de la guerra del Arauco, al pasar al Tucumán a tomar su gobierno llevó consigo como su hueste y compañía muchos de sus soldados, capitanes y sargentos que, a su vez, lo habían acompañado en Flandes. Sobre Juan de Betanzos, sabemos que fue otra persona relacionada con Alonso de Ribera, ya que este le otorgó en 1616 una merced de tierras en Colchagua, Chile. (DE RAMÓN, Armando "Los censos y el desarrollo agrícola de la región central de Chile durante el siglo XVII. Una hipótesis de trabajo", Historia, núm. 16, Santiago de Chile, 1981, p. 178).
} 
[...] porque quando se quitaron no avian acavado de visitar sus distritos." 19 Posteriormente en carta de 11 de febrero de 1608, Ribera incluirá la información brindada por el Teniente del Partido de la Ciudad de Tucumán, Diego Fernández de Cordova. ${ }^{20}$ Por todo lo expuesto, hipotetizamos que el Teniente del Partido de la ciudad de Tucumán habría sido nombrado de manera posterior a los de Santiago del Estero y de Córdoba, teniendo poco tiempo para hacer la visita. A su vez, tanto en la primera carta como en la segunda, pareciera que para San Miguel de Tucumán solo hubiera nombrado un Teniente y delimitado un único distrito. Finalmente, en la misma carta del 11 de febrero de 1608, reitera que no le llegaron las informaciones de los Tenientes que tenían a su cargo las jurisdicciones de Salta, Jujuy, Esteco y Madrid de las Juntas, porque no pudieron realizar sus visitas.

Por estos datos, hemos ido descartando la inclusión del Partido que comprendía el valle de Catamarca en las jurisdicciones de La Rioja, San Miguel de Tucumán y Córdoba, al mismo tiempo que sostenemos que este partido fue considerado parte de la jurisdicción de Santiago del Estero, cuyos distritos habían sido los primeros en ser visitados y de los cuales resultaron los primeros informes y padrones. En este marco, podemos afirmar que el Informe de la visita corresponde al Partido de la Sierra, dentro del cual, el Gobernador Alonso de Ribera incluyó tanto la "Sierra occidental de Santiago" como el "Valle de Catamarca".

\section{El Partido de la Sierra en Santiago del Estero}

Recapitulando, la documentación de principios del siglo XVII nos indica que la jurisdicción colonial de la ciudad de Santiago del Estero comprendía tres partidos que integraban la territorialidad indígena. Nos referimos al Partido del Río Dulce; al Partido del Río Salado y al Partido de la Sierra denominados y definidos así por el Gobernador Alonso de Ribera en 1607; y considerados también por el Gobernador Felipe de Albornoz, quien expresaba en 1628 que el distrito de Santiago del Estero mantenía "tres partidos de pueblos de indios que los llaman el rio Dulce sobre que esta fundada la ciudad y el rio Salado y Sierra." 21

En tal sentido, creemos que tanto las antiguas diferenciaciones espaciales y ambientales intuidas por los primeros cronistas que también podían marcar diferencias entre las sociedades indígenas del río Dulce, del Salado y de la Sierra, como las distintas apropiaciones y usos de los espacios que los actores iban haciendo, fueron usadas por estos funcionarios coloniales para identificar los espacios de las encomiendas tributarias a la ciudad de Santiago del Estero. Nos parece importante recuperar estas definiciones, en tanto la jurisdicción de Santiago fue bastante móvil en relación con otras (como la de Córdoba que mantuvo los términos de su distrito más estables), incorporando en épocas tempranas encomiendas que, para fines del siglo XVII, pasarían a ser parte de

\footnotetext{
${ }^{19}$ Carta de Alonso de Ribera al Rey del 14 de mayo de 1607. AGI. CHARCAS, 26,R.8,N.46. (Transcripción completa en CARMIGNANI, Leticia "Las visitas de los Tenientes...", cit., p. 12)

${ }^{20}$ Carta de Alonso de Ribera al Rey del 11 de febrero de 1608. AGI. CHARCAS, 26,R.8,N.46. (Transcripción completa en, CARMIGNANI, Leticia“Las visitas de los Tenientes..." cit., pp. 1324)

${ }^{21}$ Carta del Gobernador de Tucumán, don Felipe de Albornoz, al rey de España, 1628, en: JAIMES FREYRE, Ricardo El Tucumán colonial (Documentos y mapas del Archivo de Indias), Vol. I. Imprenta y Casa Editora de Coni Hermanos, Buenos Aires, 1915, p. 159.
} 
las jurisdicciones de San Fernando del Valle de Catamarca, de San Miguel de Tucumán o de La Rioja, pero cuyas historias previas deben rastrearse en los documentos referidos a la cabecera de la Gobernación.

Hasta el momento, asociábamos la Sierra de Santiago del Estero a la de Guasayán y a la vertiente oriental de Ancasti, y entendíamos que el valle de Catamarca en su sector norte correspondía a la jurisdicción de San Miguel de Tucumán y en su sector sur, o valle de los Capayanes, a La Rioja, siendo Choya (o la confluencia de los ríos del Tala y del Valle) el punto por donde pasaba la línea divisoria. ${ }^{22}$

Es por esto que el valle de Catamarca se nos presenta como un espacio difícil de integrar unívocamente a una u otras de las jurisdicciones, a lo que se suma el hecho de que en 1600 el obispo Trejo lo consagró como doctrina particular (integrando todo el valle desde Paclin hasta los Capayanes) y de que las mercedes de tierras y de encomiendas del valle de Catamarca fueron otorgadas a vecinos de las ciudades de La Rioja, San Miguel, Londres y Santiago del Estero. Asimismo, las vecindades de sus titulares fueron cambiando a lo largo del período colonial hasta la fundación de San Fernando en 1683, lo que conlleva para la práctica investigativa que la búsqueda documental de información sobre la región sea particularmente difícil debido a que, hasta 1683, no existió un cuerpo capitular que concentrara el registro y la documentación de las actividades de los colonos españoles con intereses en el valle, propietarios y/o encomenderos; como tampoco contamos con visitas $\mathrm{u}$ otro registro colonial de la población del espacio de manera específica para la primera mitad del siglo XVII. ${ }^{23}$

Aclarado esto, analicemos entonces cuál es la novedad que aporta el documento que aquí recuperamos. En primer lugar, el Informe comienza diciendo que "hasta agora que dado buelta a mi distrito", detallando más adelante que visitó a "los yndios desta sierra y valle", lo que nos permite suponer que el distrito del Teniente incorporaba tanto la Sierra como el Valle.

En segundo lugar, fue firmado en Alivigasta -en "esta sierra"- y Francisco de Betanzos señala que "ayer bine del valle de Catamarca a esta sierra"y que "avia andado por aqui yo, antes que passara al valle". Es decir, puede suponerse que el Teniente hizo su visita saliendo de Santiago del Estero, recorriendo la sierra propiamente dicha y luego, pasando al valle de Catamarca, espacio que también conformaría su partido, regresando a la sierra para concluir la visita en Alivigasta, antes de regresar a Santiago del Estero.

Con respecto al espacio, cuya principal referencia es el valle de Catamarca, la descripción del Teniente es la siguiente:

\footnotetext{
${ }^{22}$ La jurisdicción de la ciudad de La Rioja establecida por el Gobernador Juan Ramírez de Velasco terminaba "camino de la ciudad de San Miguel del Tucumán, hasta el pueblo, hacienda y valle de Choya" (en LARROUY, Antonio "Los indios del Valle de Catamarca. Estudio histórico", Revista de la Universidad de Buenos Aires, Año XI, Tomo XXVII, Imprenta Coni Hermanos, Buenos Aires, 1914, p. 173).

${ }^{23}$ Un estudio sobre el proceso de definición de la jurisdicción de Catamarca: BRIZUELA DEL MORAL, Felix y ACUÑA, María Isabel "La jurisdicción territorial de Catamarca", Congreso Regional de Ciencia y Tecnología NOA 2002, UNCa, Catamarca, 2002:

http:// www.editorial.unca.edu.ar/Publicacione $\% 20$ on $\% 20$ line/CD

\%20INTERACTIVOS/NOA2002/Jurisdiccion\%20Territorial\%20Catamarca.pdf. Otro, desde una perspectiva etnohistórica: SCHAPOSCHNIK, Ana "Cuando Catamarca aún no era Catamarca. Etnohistoria de Colpes (siglos XVI y XVII)", Población y Sociedad, 4, 1996, pp. 135-156: http://www.poblacionysociedad.org.ar/archivos/4/P\&S-V4-Schaposchnik.pdf
} 
"Es un valle entre dos sierras las más altas que hay en la Gobernación que casi están norte sur y así corre el valle.

La una es la de Londres [Ambato] que porque aquella parte que cae al valle no se puede andar ni comunicar con los indios de Londres, si no es con mucha dificultad por el mal camino que hay $[\ldots]$

“... se enbeve el agua del rio y se aparta la una sierra [Ambato],la vuelta de La Rioja y la otra [Ancasti], la vuelta de Córdoba y ensanchan allí tierra despoblada [...] [aproximadamente desde Capayan, hacia el sur]"

"En cuatro pueblos que hay desdedonde se juntan las sierras de Londres [Ambato] y ésta [Ancasti-Alto] con otra sierresita para arriba [Gracian],que aunque es doctrina del valle hace angostura y luego ensancha dicho valle arriba hasta el pueblo de Gines de Lillo [Singuil]que todo se entitula Catamarca,que aunque he dicho a Vuestra Señoria no haber más de doce leguas, se entiende de lo angosto [hacia] abajo, que de allí[hacia] arriba hay otras catorce de mala tierra de andar porque se pasa un río veinte y dos veces para ir a estos cuatro pueblos y con las muchas aguas de este año no he podido passar [...] y ahora he de entrar por la parte de Tucuman [cuesta de Paclin] porque el dicho rio no se puede andar parte de abajo..." 24

\section{Mapa I: La Sierra de Santiago y el Valle de Catamarca}

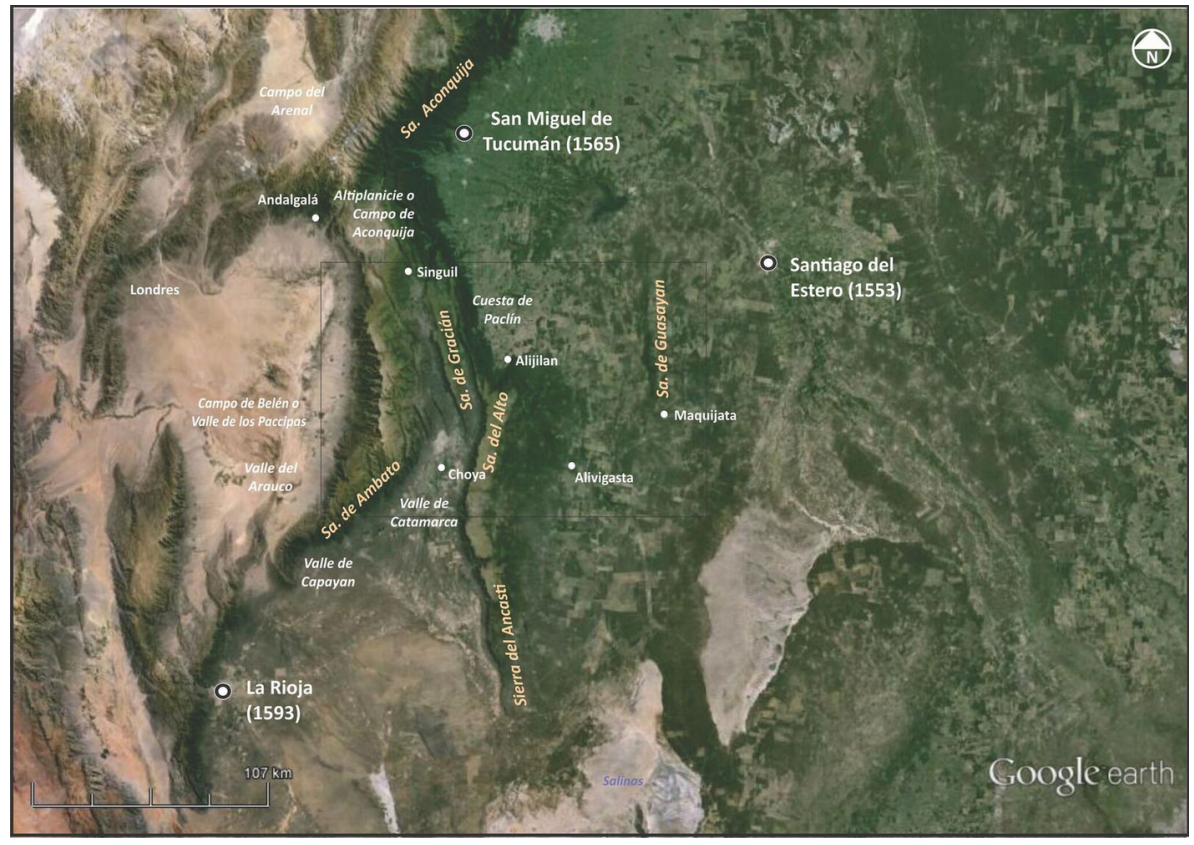

${ }^{24}$ ABNB, CACh, 580, folios 1r.-4v. Transcripción del Informe de las mismas autoras, en este mismo número de la Revista. Todos los resaltados y los agregados entre corchetes, nos pertenecen. 


\section{Mapa II: Los tres sectores del Partido de la Sierra descriptos por Francisco de Betanzos en 1607}

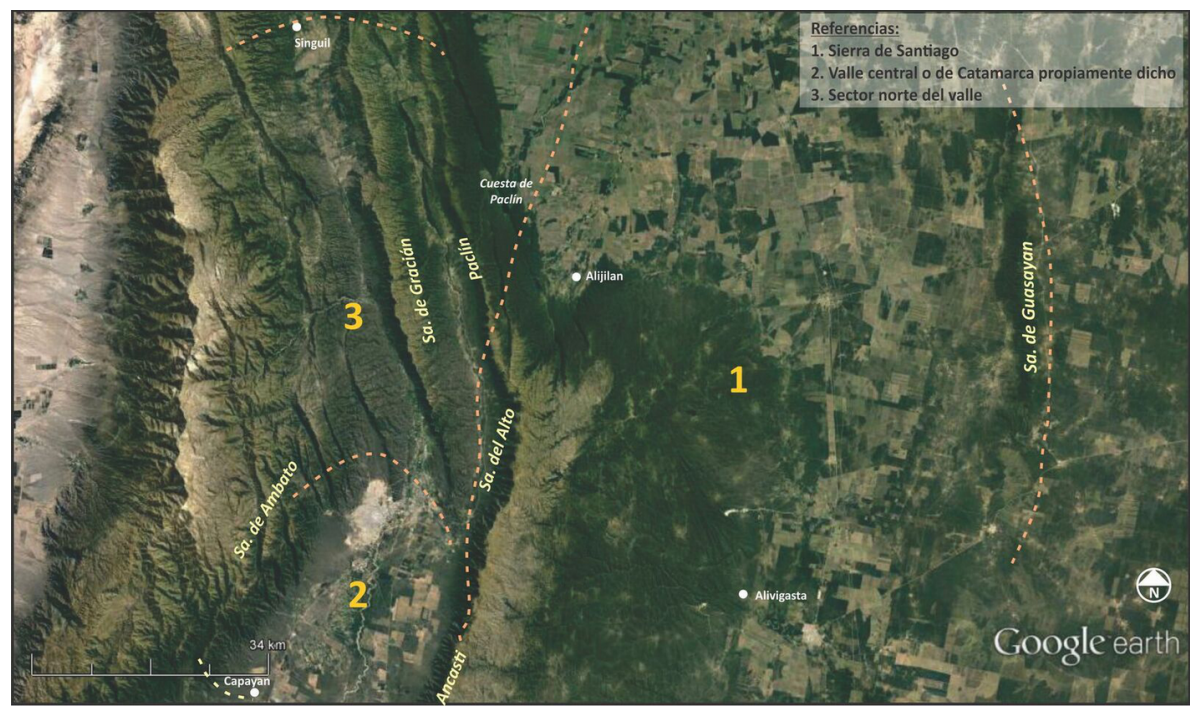

Una lectura atenta del documento, al cual le hemos ido agregando las referencias geográficas necesarias para su comprensión, permite inferir que el Teniente diferencia geográficamente, tres espacios.

En primer lugar, "Londres" que designa tanto la sierra del Ambato como el espacio y los grupos que se asentaban en la falda occidental del Ambato y se extendía por los salares y el Campo de Belén o Valle de los Paccipas hacia el oeste y hacia el norte hasta lo que el fuerte de Andalgalá. Este espacio no fue visitado por Betanzos, ya que, en ese momento, el valle de Londres pertenecía a la jurisdicción de La Rioja y los indios a sus vecinos encomenderos y, por lo tanto, fue visitado por otro Teniente del Gobernador Ribera, designado para ese distrito. ${ }^{25}$

En segundo lugar, el "valle de Catamarca" al que el informante piensa y representa en su discurso en dos subregiones: a) el valle central o de Catamarca propiamente dicho, desde el valle de los Capayanes 12 leguas hacia el norte, es decir desde donde "se aparta la una sierra [de Ambato y da] la vuelta de La Rioja y la otra [de Ancasti] la buelta a de Cordova y ensanchan alli tierra despoblada"; extendiéndose hacia el norte para encajonarse al confluir los cordones de Ambato, el Alto y Gracián [La Puerta], donde comienza la segunda subregión: b) lo que Betanzos describe como "catorce [leguas]de mala tierra de andar" que es donde el valle "hace angostura y luego ensancha dicho valle

\footnotetext{
${ }^{25}$ Hasta 1607, que se re-fundó Londres como San Juan Bautista de la Ribera, el valle de Londres era un partido o distrito asignado a un Teniente de natural, de la jurisdicción de la ciudad de La Rioja. (CARMIGNANI, Leticia "Las visitas de los Tenientes...", cit., p. 4). El amplio espacio del oeste del Ambato, asimismo, se corresponde a dos subregiones. En primer lugar, los asentamientos indígenas ubicados en la falda occidental de la sierra, limitados por el salar de Pipanaco: Mutquin, Sijan, Saujil, Pisapanaco, Colpes, Pipanaco; y aquellos ubicados más al norte, en las estribaciones meridionales del cordón de Aconquija en los distintos valles y oasis como Andalgalá y Gualfín, e incluso sería precisamente el espacio de contacto y bisagra con el valle de Yocavil y Calchaquì, al sur de Campo del Arenal. Los grupos indígenas de ambas zonas, a los que se sumaron aquellos de los valles dependientes de La Rioja estuvieron comprometidos y llevaron adelante la resistencia calchaquí del período 1630-1643. Un estudio particular de Londres y la producción de sus encomiendas, para el mismo período en: QUIROGA, Laura "Las granjerías...", cit.
} 
arriba hasta el pueblo de Ginés de Lillo [Singuil]", aclarando que en tiempos de lluvia solo puede accederse a ese sector del valle desde Tucumán, desde la cuesta de Paclín, y no desde el valle de Catamarca propiamente dicho. ${ }^{26}$

Finalmente, el espacio de "la Sierra" que se correspondería a la vertiente oriental del cordón Ancasti-Alto-Gracián. Este espacio comienza en la ladera oriental del cordón Alto-Ancasti y su piedemonte y va descendiendo hasta el Río Dulce, interrumpido por la sierra de Guasayán, ha sido denominado por Constanza Taboada como "tierras bajas catamarqueñas" y asociado al Partido de la Sierra. ${ }^{27}$

El padre Larrouy ya señalaba la dificultad para nominar este espacio, diciendo que

“entre las cadenas de montañas de la República Argentina, la que forma el lado Este del Valle de Catamarca es verdaderamente singular, no por su extensión, unos 200 kilómetros, ni por su altura que no pasa de 2000 metros sobre el nivel del mar, y 1500 sobre el del Valle, sino por otras circunstancias. Por el pronto, no tiene ningún nombre general. En Catamarca, decimos 'la Sierra' [...] para designarla, los textos de geografía tienen que valerse de circunlocuciones: escriben Sierra de Ancasti y Alto, y agregan que más al Norte se llama también de Guayamba, del Totoral y de Balcosna [...] La misma multiplicidad de nombres para un solo objeto demuestra que no lo tiene propio." 28

Larrouy destacaba que otra característica de este cordón es que mientras en su vertiente occidental se presenta como una "muralla", "la [vertiente] opuesta declina insensiblemente hasta confundirse con la llanura, a 60 kilómetros de la cumbre en algunas partes. Se comprende, pues, que no se encuentre ninguna población en la falda que cae al valle de Catamarca, pero

\footnotetext{
26 Ana Schaposchnik señaló, sobre la denominación valle de Catamarca, usando el mismo documento junto con cédulas de encomiendas, que "en la topografía de la época, tal denominación indicaba el territorio que iba desde La Puerta hacia el sur: éste habría sido el Valle de Catamarca propiamente dicho", señalando que el sector norte, de La Puerta hasta Singuil era otro valle diferenciado del de Catamarca y que podría ser el de Colpes, pero entendido no a nivel geográfico sino a nivel de organización social o política, es decir, como parte de un espacio controlado por una misma jefatura y que integraría las dos vertientes del Ambato en dicho sector. SCHAPOSCHNIK, Ana "Cuando Catamarca...", cit., pp. 142-143. No estamos contradiciendo lo señalado por la autora, la diferencia es que desde nuestra lectura "jurisdiccional y geográfica", el espacio que "todo se intitula Catamarca" integra el valle desde Singuil al norte hasta Capayan -aproximadamente- al sur. Esta lectura más amplia del documento, no está reñida con espacios indígenas y prehispánicos de articulación política que excedan o se superpongan con las delimitaciones y jurisdicciones de la dominación colonial y que podrían desprenderse del análisis de otro tipo de fuentes como son las cédulas de encomienda.

27 TABOADA, Constanza “Repensando la Arqueología de Santiago del Estero. Construcción y análisis de una problemática”, Relaciones, XXXVI, 2011, pp. 197-219: http://www.saantropologia.com.ar/wp-content/uploads/2015/01/Relaciones

\%2036/Relaciones\%20XXXVI.pdf y TABOADA, Constanza "El temprano en Santiago del Estero y las tierras bajas de Catamarca. Problemáticas y particularidades", Encuentro Arqueología del Período Formativo en Argentina, Tafí del Valle, 2012: http: / / www.ises.org.ar/arqueologia//pdf / 2012319227192614.pdf

28 LARROUY, Antonio "Formación de la Provincia"; "Colonización de la Provincia"; "Documentos Inéditos de la época colonial. 1607-1808", en LARROUY, Antonio y SORIA, Manuel (dir.) Autonomía Catamarqueña. Homenaje en su primer Centenario, Talleres Peusser, Buenos Aires, 1921, p. 35.
} 
que hay muchas en la oriental, departamentos de Alto y Ancasti especialmente." Larrouy coincide en que "la Sierra de Santiago" comprendía tanto la sierra Ancasti-Alto y su piedemonte oriental como la sierra de Maquijata. "Esta fue la que dio a toda la Sierra su nombre primitivo de Sierra de Santiago, pues pertenecía a aquella jurisdicción." ${ }^{29}$

Amalia Gramajo había advertido sobre los pueblos de indios de las "Sierras del Oeste", que los mismos pertenecieron a la jurisdicción santiagueña hasta la fundación de la ciudad de San Fernando:

“La posesión catamarqueña se hizo efectiva después de las tareas de deslinde y amojonamiento llevadas a cabo entre 168485, de modo que allí quedaron en su interior y a espaldas del antiguo carril que va por su ladera oriental y pie de las Sierras, varios de esos pueblos de indios mencionados: Tavigasta, Collagasta, Alijilán, Sicha, Yocavil, Alivigasta, Simogasta, anteriormente todos de la jurisdicción de Santiago del Estero".$^{30}$

En síntesis, el análisis de este documento en 1607 a tono con los límites de la Doctrina del Valle establecida en 1600 por el obispo Trejo que consideran todo el valle y no su división norte-sur (ciudades de San Miguel-La Rioja); nos permitiría asegurar que el Gobernador Alonso de Ribera tuvo la intención de incorporar en un solo distrito o partido denominado "de la Sierra" no solo la vertiente oriental del Alto-Ancasti que era jurisdicción de la ciudad de Santiago, sino también el valle de Catamarca desde el valle de los Capayanes al sur hasta el pueblo de Singuil, al norte.

\section{Hacia una nueva regionalización}

Más allá de estas definiciones jurisdiccionales, entendemos que otro aspecto importante del documento es que nos permite plantear una nueva regionalización en función de las encomiendas y sus producciones; pudiendo demostrar que muchas de las mercedes de tierras y de encomiendas otorgadas en el valle de Catamarca se complementaban, por el tipo de recursos extraídos o producidos, con otros espacios de la región del Tucumán. Analicemos entonces, la información que nos brinda sobre los recursos.

El Teniente Francisco de Betanzos diferencia en 1607las subregiones del Partido de la Sierra: valle de Catamarca propiamente dicho, valle en su sector norte y Sierra, por el tipo de recursos y de actividad económica principal. En el valle central o de Catamarca, propiamente dicho, "todo el trato es algodon" y son de central importancia para la economía indígena los algarrobales; en los pueblos del sector norte del valle hasta el pueblo de Singuil "no se coge algodon hay cria de mulas y ganados, acuden a hilar y a otras granjerias, tierra de muchas papas y muy buen maiz y mucho ganado de la tierra".Finalmente, en la sierra "es el trato lienço y alpargatas cordovanes" y guascas de chaguar, a pesar que sabemos que también se produce algodón, ya que en la encomienda de Luis de Gallegos, en Alivigasta "dan mucho fructo treinta yndios que alli tiene se cojeran este año dos mill arrobas de algodón”, según el mismo Betanzos.

\footnotetext{
${ }^{29}$ LARROUY, Antonio “Formación de la...", cit., p. 36.

${ }^{30}$ GRAMAJO de MARTÍNEZ, Amalia "Pueblos de Indios Postconquista de la Jurisdicción de Santiago del Estero. Investigación en las Fuentes", Relaciones de la Sociedad Argentina de Antropología XVIII, Buenos Aires, 1990-92, p. 203.
} 
Si nos detenemos en el algodón, claramente el Teniente nos alerta acerca de la subregión que lo produce: correspondería al valle de Catamarca propiamente dicho, donde luego se asentó la ciudad de San Fernando y donde muy tempranamente se instalaron haciendas españolas con algodonales explotados por la mano de obra de las encomiendas y bajo el sistema de servicios personales; en menor medida, pero también sabemos que existía algo de producción de algodón en la Sierra. ${ }^{31}$

Sobre el valle de Catamarca propiamente dicho, la producción se asentaba en las encomiendas y núcleos productivos españoles con mano de obra indígenas. Allí se asentaban las encomiendas de Motimo o Motimogasta que incorporaba las tierras de Polco y Choya, entre otras-, las propiedades originadas en las mercedes de Autigasta, Guaycama, Collagasta, Villapima. En la zona norte del valle, la producción principal sería la ganadería y suponemos que, en ese sentido, el hilado podría ser de lana, aunque no descartamos que también hayan podido hilar y producir piezas de lienzo, obteniendo el algodón producido en el sector central del valle.

Por la vertiente oriental de la Sierra Alto Ancasti, donde se asentaban los pueblos de indios de Alijilan, Tavigasta, Figasta, Sicha, Icaño y las estancias y mercedes de Quimilpa, Alivigasta y Obanta, entre otras, y en la Sierra de Guayamba, la de Maquijata; Betanzos señala que se producen cordobanes, guascas de chaguar y lienzo, indirectamente también nos dice que se produce algodón, pero como ya señalamos hasta el momento solo podemos corroborarlo en Alivigasta.

Es decir, podríamos hipotetizar que, además de la recolección del chaguar, propia de esta subregión y la producción textil algodonera en algunos sitios, la actividad principal de la Sierra era la transformación de las materias primas producidas en el valle de Catamarca en sus dos sectores (algodón, para la confección de lienzos y ganadería, para la elaboración de cordobanes); así como la elaboración de alpargatas gracias al chaguar de la misma región. Veremos a continuación, como el valle de Catamarca y la Sierra de Santiago serán el espacio complementario de la producción de otras regiones como el Partido del Salado o la jurisdicción de San Miguel de Tucumán.

Los encomenderos que cita el Informe de Betanzosson ${ }^{32}$, en el valle central: Juan Bautista Muñoz (Autigasta y Guaycama ${ }^{33}$ ), Luis de Medina (Choya), ${ }^{34}$ Pedro de Maidana (Motimo), ${ }^{35}$ en el norte del valle: Gines de Lillo

\footnotetext{
${ }^{31}$ La información con la que contamos al momento no nos permite precisar los sitios o subregiones en la Sierra donde se producía algodón, a excepción de la referencia que acabamos de reseñar sobre el pueblo de indios de Alivigasta, a lo que sumamos la información acerca de que la encomienda de Maquijata, a pesar de la misma jurisdicción, no producía algodón, sino que lo compraba y uno de sus proveedores era Luis de Gallegos (que, como ya veremos, era el encomendero de Alivigasta). Sobre Maquijata, ver FERREIRO, Juan Pablo "Maquijata. Encomienda, tributos y sociedad en el Tucumán colonial temprano", en LORANDI, Ana María El Tucumán colonial y Charcas, Vol. 2, FFyL-UBA, Buenos Aires, pp. 73-128.

${ }^{32}$ Esta lista no excluye otros encomenderos de la región no citados por el visitador, por ejemplo, Nuño Rodríguez Beltrán de Pomagasta (actual La Puerta), importante centro algodonero, (LIZONDO BORDA, Manuel Documentos coloniales relativos a San Miguel de Tucumán y a la gobernación de Tucumán. Siglo XVII, Tomo II, publicaciones de la Junta Conservadora del Archivo Histórico de Tucumán, Tucumán, 1937, p. 253)

${ }^{33}$ Sabemos que los pueblos de Guaycama de Muñoz producían una importante cantidad de algodón. (LIZONDO BORDA, Manuel Documentos coloniales..., cit., pp. 103-104.)

34 Juan Bautista Muñoz y Luis de Medina eran encomenderos de la ciudad de San Miguel de Tucumán.

${ }^{35}$ Pedro de Maidana era vecino encomendero de La Rioja.
} 
(Singuil), ${ }^{36}$ Diego Granero (Paclin); ${ }^{37}$ y en la vertiente oriental de la Sierra: Luis de Gallegos (Alivigasta) y García Barata (Sichagasta). ${ }^{38}$

Tomemos el ejemplo del encomendero, vecino de Santiago, García Barata, quien en 1608, responde a un censo declarando que "Llámase mi repartimiento Lonsaya y Sichagasta", posteriormente afirma tener "una estancia treynta leguas desta ciudad que tendrá mil cauezas de ganado bacuno chicos y grandes, machos y hembras, y cinquenta hieguas con garañones y otras cinquenta burras y garañones, mil cauezas de ganado de cabras y obejas." ${ }^{39}$ Aunque no lo aclara, estamos en condiciones de afirmar que Lonsaya es su encomienda principal, ubicada en el Partido del Río Salado, donde además tiene su estancia, a "30 leguas de la ciudad" de Santiago del Estero; mientras que la encomienda de Sichagasta se ubicaba en la vertiente oriental de la Sierra del Ancasti, Partido de la Sierra.

Sabemos también por los Informes sobre el Río Salado, que los indios de las encomiendas del partido se ocupaban "de variadas actividades en la hacienda de su encomendero: cultivos y producción de comidas, guarda de ganado, fletes para viajes de media y larga distancia, vaquerías, construcción y obras de casas, recolección de grana, confección de alpargatas, hilado y tejido de lana" así como producción de textiles de algodón. ${ }^{40} \mathrm{~A}$ esto podríamos agregar que probablemente gran parte, o quizás casi todo, del algodón que se hilaba y tejía en el Salado provenía de encomiendas asentadas en otras regiones ambientales productoras de algodón, como es el caso del valle de Catamarca. ${ }^{41}$ Además, podía sumarse el trabajo a gran escala del chaguar o

\footnotetext{
${ }^{36}$ En 1607, al refundar Londres como San Juan Bautista de la Ribera, Ginés de Lillo como encomendero de Sabuil/Sajuil pasará a ser vecino de la recién fundada ciudad. Ver QUIROGA, Laura "Las granjerías...", cit., p. 14.

${ }^{37}$ Diego Granero de Alarcón, vecino de San Miguel, recibió la merced de tierras de Paclin.

${ }^{38}$ Luis de Gallegos y García Barata ambos, vecinos de Santiago del Estero. “Descripción general de Santiago del Estero. 9 de octubre de 1608", en PÉREZ SÁEZ, Vicente y OSÁN de PÉREZ SÁEZ, María Fanny El Español de la Argentina. Documentos para su Historia (Noroeste). Volumen I. Consejo de Investigación Universidad Nacional de Salta, Salta, 1997.

${ }_{39}$ "Descripción general de Santiago del Estero. 9 de octubre de 1608”, en PÉREZ SÁEZ, Vicente y OSÁN de PÉREZ SÁEZ, María Fanny El Español..., cit., pp. 63-64.

${ }^{40}$ CASTRO OLAÑETA, Isabel "La Numeración de los indios...", cit., p. 10. La lectura atenta de este documento nos ha permitido revisar y precisar este planteo que hipotetizaba la producción de algodón en el Salado. Una aclaración que debemos hacer aquí es que, hasta el momento, no hemos podido comprobar la producción de algodón en las encomiendas del Salado ya que no contamos con los Informes originales de los Tenientes de Naturales del Salado y del Dulce como el caso de la Sierra- sino un resumen general de los tres partidos que integra las apreciaciones de Ribera sobre toda la jurisdicción. (Ver carta completa en CARMIGNANI, Leticia "Las visitas de los Tenientes...", cit., pp. 1-4)

Lo que este nuevo Informe nos permite corroborar es que había encomenderos que poseían indios en distintos distritos, como el Salado y la Sierra, logrando articular el trabajo indígena de ambos espacios dedicándolo al cultivo en el valle de Catamarca y al hilado y tejido en el Salado, como es el caso de García Barata con Lonsaya y Sichagasta.

${ }^{41}$ Asimismo, nos preguntamos si las denunciadas malocas llevadas adelante por García Barata desde la Sierra a los indios del valle de Catamarca no tenían como objetivo robar mano de obra preparada y con habilidades para el trabajo con el algodón y de la cabuya e, incluso, robar lo producido. No solo el Informe de Betanzos nos permite pensar la cotidianeidad de las malocas en la Sierra, sino también algunos planteos de Ferreiro sobre las malocas realizadas por orden del encomendero de Maquijata, en la misma región, y pagadas a un mercenario. (FERREIRO, Juan Pablo Maquijata. Encomiendas, tributo..., cit., p. 119)

La pregunta sobre las habilidades indígenas para el cultivo y trabajo del algodón nos lleva a indagar acerca de su existencia en tiempos prehispánicos, un tema que está actualmente en plena discusión. En este artículo no abordaremos el problema de los grupos que habitaban la región antes de la llegada de los españoles, sus características y sus formas de organización
} 
cabuya para la elaboración de alpargatas, cinchas y bolsas no solo en el Salado, sino también en el Dulce, proveniente, quizás en parte, de la Sierra del oeste santiagueño. ${ }^{42}$

Otro ejemplo de complementariedad del valle de Catamarca con otra región del Tucumán, es el caso de dos encomenderos ya mencionados, Juan Bautista Muñoz y Luis de Medina, quienes además de contar con encomiendas situadas en el corazón del valle catamarqueño con una importante producción algodonera, habían adquirido tierras en la jurisdicción de San Miguel donde tenían la vecindad, en la región de Belicha y Gastona: "En 1579 Juan Bautista Muñoz solicita tierras en las márgenes del río Guaycombo (hoy Gastona) en los siguientes términos 'por que yo tengo necesidad de una estancia para mis ganados...' Poco tiempo después Luis Medina se convertirá en vecino de Muñoz al obtener "una estancia camino real a Santiago dos leguas, más abajo de Belicha..." 43 No es casual que ambos sean "vecinos" tanto en el valle como en la región ganadera de Gastona y Belicha complementando y diversificando su producción, teniendo mercedes y encomiendas en dos espacios de producciones complementarias.

En síntesis, el valle de Catamarca fue un espacio dedicado a la producción de algodón principalmente que fue ocupado desde fines del siglo XVI y puesto en producción con los indios de encomienda, por los vecinos encomenderos de Santiago del Estero, La Rioja, San Miguel de Tucumán y Londres cuyas jurisdicciones avanzaban sobre el valle (en términos sencillos, La Rioja sobre el sector sur y central; San Miguel sobre el sector nor-este y Londres sobre el sector nor-oeste). Jurisdiccionalmente, el Gobernador Alonso de Ribera, en 1607 integró el valle al Partido de la Sierra al distrito de Santiago del Estero, creemos que en un proyecto de darle unidad jurisdiccional al mismo tiempo que hacerlo coincidir con la doctrina del valle establecida por el Obispado.

Más allá de este intento, que sabemos que no se mantendrá a lo largo de la colonia y que recién se plasmará con la fundación tardía de la ciudad de San Fernando en 1683; entendemos que lo importante a destacar es que existe una lógica regional de producción que permite pensar en una complementariedad de las propiedades españolas y de las encomiendas donde el Valle y la Sierra

sociopolítica, lo que queda pendiente para un próximo estudio. Una síntesis acerca de los argumentos que sostienen que el cultivo del algodón en el Tucumán era prehispánico o que fue introducido por los españoles, (las autoras sostienen la segunda hipótesis): LEMA, Verónica y CAPPARELLI, Aylen "El algodón (Gossypium sp.) en el registro arqueológico del NOA: su presencia pre y post hispánica" en MARCONETTO, Bernarda, BABOT, Pilar y OLISZEWSKI, Nurit (eds.) Paleoetnobotanica del Cono Sur: estudios de casos y propuestas metodológicas, Editorial Ferreyra, Córdoba, 2007, pp. 69-78. Una reciente investigación con nuevos avances al respecto, en TABOADA, Constanza, LOPEZ CAMPENY, Sara y ANGIORAMA, Carlos “A propósito de una placa de metal con un tejido de algodón adherido identificados en el Museo Wagner de Santiago del Estero (Argentina). Usos, contextos e implicancias en relación a procesos locales, incaicos y coloniales", Estudios Atacameños, en prensa. Sobre Santiago del Estero, ver los trabajos de TABOADA, Constanza "Repensando la Arqueología...", cit., pp. 197-218; FARBERMAN, Judith y TABOADA, Constanza "Las sociedades indígenas del territorio santiagueño: apuntes iniciales desde la arqueología y la historia. Período prehispánico tardío y colonial temprano", Runa, vol. 33, núm. 2, Universidad de Buenos Aires, Buenos Aires, 2012, pp. 113-132.

${ }^{42}$ La producción de alpargatas de chaguar o cabuya en el Dulce, también era de especial importancia, especialmente en las encomiendas reales de Soconcho y Manogasta. (FARBERMAN, Judith "Recolección, economía campesina y representaciones de los montaraces en Santiago del Estero, siglos XVI a XIX", Prohistoria, año X, núm. 10, Rosario, 2006, p. 18).

${ }^{43}$ NOLI, Estela "Algarrobo, maíz y vacas. Los pueblos indios de San Miguel del Tucumán y la introducción de ganados europeos (1600-1630)", Mundo de antes, núm. 1, Instituto de Arqueología y Museo, Universidad Nacional de Tucumán, Tucumán, 1998, p. 39. 
complementan sus especialidades con aquellas de otros espacios y que creemos debe continuar estudiándose.

El Partido de la Sierra y sus subregiones -Sierra Ancasti-Alto-Gracián y Valle de Catamarca-corresponden tanto a jurisdicciones coloniales dentro de un marco administrativo-territorial, pero, por otra parte, deben ser comprendidas en el marco de la espacialización que los agentes hacen de estas jurisdicciones. En este caso, hemos abordado este problema en relación a la complementariedad productiva de los espacios cruzando la información sobre la vecindad de los encomenderos y propietarios, los límites jurisdiccionales de cada ciudad e incluso los límites establecidos por el obispado en su definición de las doctrinas, para ver superposiciones, complementariedades y uso del espacio en un período en el cual la Gobernación del Tucumán se está aún definiendo y continúa en un largo proceso de consolidación y de conquista de territorios rebeldes.

Darío Barriera propone que las primeras definiciones jurisdiccionales de las Gobernaciones de mediados de siglo XVI al Sur de Charcas, ignoran la realidad del terreno que delimitan y son los actores, quienes desde la conquista van diseñando un recorrido más acorde al terreno y a las posibilidades. Así, el "proceso de espacialización", o el modo real en que el territorio se convertía en un espacio, "se realizaba sobre los ejes de la comunicación y las condiciones de accesibilidad en sentido amplio." ${ }^{44}$ De esta manera, mientras en la teoría se describían jurisdicciones, los actores conocían y manejaban otros conjuntos espaciales, otros factores limítrofes y otros espacios de circulación que no siempre coincidían con dichas delimitación.

En este marco, entendemos que para principios de siglo XVII, la especialización productiva regional, organizada en torno a las encomiendas de indios fue uno de los factores que contribuyó al proceso de espacialización de estas jurisdicciones. Esto permitió que para el momento analizado, el espacio del Valle y la Sierra, no se encontrara determinado solamente por la jurisdicción de una ciudad, sino que en él, los actores fueran haciendo confluir encomiendas de vecinos de diferentes ciudades, primando una especialización productiva regional que sería complementaria a otros espacios de encomienda. Este artículo ha pretendido avanzar en esta línea de estudio proponiendo algunas ideas y revisando otras, al mismo tiempo que dejamos abiertas varias preguntas para seguir trabajando en el mismo sentido.

Isabel Castro Olañeta

Leticia D. Carmignani

Córdoba, Argentina, mayo de 2017

${ }^{44}$ BARRIERA, Darío Abrir puertas..., cit., p. 32. 